and Dr. Jules Carret has just published an ingenious book on the subject. ${ }^{1}$

The paper, of which the following is an abstract, is an attempt to investigate the results of the supposition that the earth is slowly changing its shape from internal causes. The first part is devoted to the mathematical consideration of the precession and nutations of a spheroid slowly undergoing such a change. It is shown that the obliquity of the ecliptic must have remained sensibly constant throughout geological history, and that even gigantic polar icecaps cannot have altered the position of the Arctic circle by so much as three inches; and this would be the most favourable redistribution of matter for producing that effect.

But a slow distortion of the earth would displace the principal axis of figure of the earth, and the axis of rotation would always sensibly follow the axis"of figure. Thus the result would be a change in the geographical position of the poles, without any alteration of the diameters of the arctic circles, or in the width of the tropics.

For reasons, which cannot be given here, it is maintained that the earth would not be rigid enough to resist the effects of considerable departures from the figure of equilibrium, such as would arise from a wandering of the pole of figure from its initial position ; and that readjustments to an approximate form of equilibrium would probably take place, at considerable intervals of time, impulsively by means of earthquakes. Such periodical adjustments would not sensibly modify the geographical path of the principal axis as due to terrestrial deformation.

The rest of the paper is given to the consideration of the kinematical question of the change in the geographical position of the pole, due to any distortion of the earth. It is assumed, in the first place, that the deformation is such that there is no change in the strata of equal density; and accordingly all suppositions as to the nature of the internal changes accompanying geological upheaval and subsidence are set aside. The forms of continents and depressions are investigated, which would cause the maximum deflection of the pole for elevations and depressions of given amounts.

In order to make numerical application to the case of the earth, some estimate is required of the extent to which it may have become distorted during any one geological period. From the consideration of certain facts, the author believes that from $\frac{1}{10}$ to $\frac{1}{2} \delta$ of the whole earth's surface may have, from time to time, undergone a consentaneous rise or fall ; and that the vertical rise or fall may be about 10,000 feet, or rather equivalent to about I0,000 feet, when allowance is made for the influx of the sea into depressed areas.

The first application given in this paper is to continents and seas of the most favourable shapes and positions. It may be here stated that if $\frac{1}{00}$ of the earth's surface is elevated by 10,000 feet, the deflection of the pole is II $1^{\prime} ;$ if $\frac{1}{20}$ of the whole surface, $\mathrm{I}^{\circ} 46 \frac{1}{2}^{\prime}$; if $7^{\frac{\pi}{0}}$, $3^{\circ} 17^{\prime}$; and if $\frac{1}{2}, 8^{\circ} 4 \frac{1^{\prime}}{2},{ }^{2}$ In each case an equal area is supposed to fall simultaneously.

Other examples are also given for continents and seas which do not satisfy the maximum condition; in some the boundaries are abrupt cliffs, in others shelving.

The conclusion is arrived at that a single large geological change, such as those which obtain on the earth, is competent to produce an alteration in the position of the pole of from one to three degrees of latitude, on the hypothesis that there is no change in the law of internal density.

Various other hypotheses as to the nature of the internal changes accompanying the deformation of the earth are discussed.

I. It is shown that if upheaval and subsidence are due

I "Le Déplacement Polaire." Savy, Paris, 1877 . America about "033 of
2 The area of Africa is about "059, and of South Amera the earth's surface. to a shrinking of the earth as a whole, but to the shrinking being quicker than the mean in some regions and slower in others, the results are the same as those previously attained.

2. The increase of surface-matter due to the deposit of marine strata also gives the same results.

3. The hypothesis that upheaval and subsidence are due to intumescence or contraction immediately under the regions in question is considered. Under certain special assumptions, too long to recapitulate, it is shown that the previous results must be largely reduced. It appears that if the swelling or contracting stratum is tolerably thin and at all near the surface, the deflection of the pole is reduced to quite an insignificant amount. Even if the intumescence extends right down to the centre of the earth in a cone bounded by the elevated region, the results would be only about $\frac{2}{3}$ of the former ones. Hence it appears that the earlier results can only be stated as the greatest possible for given superficial changes.

In conclusion it is pointed out that if the earth be quite rigid, no redistribution of matter in new continents could ever cause the deviation of the pole from its primitive position to exceed the limit of about $3^{\circ}$. But if the previously maintained view is correct, that the earth readjusts itself periodically to a new form of equilibrium, then there is a possibility of a cumulative effect; and the pole may have wandered some $10^{\circ}$ or $15^{\circ}$ from its primitive position, or have made a smaller excursion and returned to near its old place. No such cumulation is possible, however, with respect to the obliquity of the ecliptic.

It is suggested that possibly the glacial period may not have been really one of great cold, but that Europe and North America may have been then in a much higher latitude, and that on the pole retreating they were brought back again to the warmth. There seem to be, however, certain geological objections to this view.

\section{THE NEW STAR IN CYGNUS ${ }^{1}$}

$\mathrm{N}$ January 9 the sky was unusually clear and the spectrum of Dr. Schmidt's Nova came out with amazing sharpness and brilliancy. In addition to the five bright lines seen on the 2 nd, two others were detected, viz. :-

No. Ia W. L. 594 Very narrow line.

, $7 \quad 4$ I4 E Excessively faint, but still certainly and repeatedly seen.

Between wave-lengths 655 and 594 the spectrum was certainly banded, and, most probably, there were two additional faint maxima of brilliancy in that interval. The continuous spectrum attains a maximum in the region about W. L. 525 , and extends, though possibly not without interruption, as far as the faint line No. 7. The star was estimated of seventh magnitude, and was of a red colour with a decided tinge of purple, reminding me forcibly of the varieties of red produced by the quartzplate in Zöllner's photometer. RALPH COPELAND

Lord Lindsay's Observatory, Dunecht, January I3

\section{OUR ASTRONOMICAL COLUMN}

THE New COMET.-.-The comet discovered by M. Borrelly, at Marseilles, on the morning of February 9, appears to have been iound independently by Herr Pechille at the Observatory of Copenhagen on the following morning. During the past week it has been making a pretty near approach to the earth, and had the weather been more favourable in Europe, it would probably have been very generally observed.

The following elements of the orbit have been calculated by Mr. Hind from the first observation by M. Borrelly, one at 